\title{
IMPROVED WEIGHT FUNCTIONS FOR STRESS INTENSITY FACTORS OF SEMI ELLIPTICAL CRACK IN STEAM TURBINE ROTOR SYSTEM
}

\author{
Damarla Kiran Prasad ${ }^{1}$, Kavuluri Venkata Ramana², Nalluri Mohan Rao ${ }^{3}$ \\ ${ }^{1}$ Gudlavalleru Engineering College, Gudlavalleru, India-521356 \\ ${ }^{2} \mathrm{~K}$. L. University, Vaddeswaram, India-522302 \\ ${ }^{3}$ Jawaharlal Nehru Technological University Kakinada, Kakinada, India-533001
}

\begin{abstract}
:
This paper presents weight function approach with piece wise polynomial interpolation function to determine the stress intensity factors at the surface and deepest points of a semi elliptical crack induced at the blade mounting locations of the rotor system. Initially, the mathematical model representing tapered rotating disc is solved and the equations for radial stress are obtained. Using the compatibility conditions, these equations are applied to plot the stress distribution at blade mounting locations in steam turbine rotor system. In the second part, weight functions with five and six terms are derived separately at the surface and the deepest points of semi elliptical crack to determine the stress intensity factors. The coefficients of these functions are determined assuming piece wise polynomial stress distribution along the crack. This weight function approach is applied to a semi elliptical crack at blade mounting locations by considering the stresses at discrete points of the crack obtained in first part and the stress intensity factors (SIFs) are determined. The results obtained are validated with the influence coefficient approach.
\end{abstract}

\section{ARTICLE HISTORY}

Received: 26.01.2019.

Accepted: 29.03.2019.

Available: 31.03.2019.

\section{KEYWORDS}

Stress intensity factor, blade mounting locations, steam turbine rotor, weight function approach, piece wise polynomial interpolation function

\section{INTRODUCTION}

The increase in demand for the thermal power generation requires effective design of components in thermal power plants. One of the critical components in the plant is turbine rotor and it is subjected to high magnitude of stresses which affects its life. The effect of failure of rotor is enormous in terms of electricity generation failure and economic loss for the plant. Hence in order to avoid the interruption of power generation and increase the reliability of turbine rotor system, it is necessary to perform the analysis of steam turbine rotor. The blade mounting location of the rotor is critical part where the stress concentration is more and increases the probability of development of crack. Most of the researchers have concentrated on examining the reasons for crack development and failure of rotor using microscopic and chemical testing on the cracked part.

Many cylindrical components having geometrical discontinuities are subjected to dynamic loading. These components may have inherent micro cracks. During the operations, these cracks may propagate and lead to failure of the cylinders. Hence determination of stress intensity factors becomes critical in these components [1-5]. The effect of geometrical discontinuities is studied by a number of researchers [6-9]. For transportation of fluids in different application, pipes are the critical components and are subjected to complex loadings. During manufacturing, defects may be incorporated in pipes which create initialization of 
cracks and the probability of failure gets worsened due to crack growth and propagation.

Banaszkiewicz et.al.[10] has analyzed nonstationary stresses and fatigue cracking in impulse steam turbine rotors and identified the most frequent locations of crack appearance. The number of cycles to crack initiation has been calculated based on the strain amplitudes correspond well with the operating experience of this type of rotors. Hattingh, D. G. et.al. [11] have presented a case study dealing with the assessment of cracking observed at steam turbine blade attachment grooves. The work outlined a fracture mechanics analysis of in service cracking observed and aimed at assessing critical defect sizes to support either replacement or repair. Banaszkiewicz, M. et.al. [12] in their work presented root cause analysis of steam turbine rotor blade groove cracking. The blades in the failed rotor are removed and it was subjected to metallographic and non destructive testing like magnetic particle testing. Based on the analysis performed, it was decided to repair two discs using the process of weld built up. The stress fields in the blade grooves were calculated and the possibility of cracking was assessed as stress corrosion. They proposed change in the blade groove geometry to ensure the tolerance to the stress corrosion cracking. Barella, S. et.al. [13] have explored the origin of the fatigue phenomenon in relation to the blade fixing method and its groove in the turbine rotor. The failed rotor was cut to open the cracked surface and the fractured surface analysis was conducted.SEM analysis along with energy dispersive spectroscopy was performed for chemical analysis. To perform the metallographic analysis, optical microscope was used for microscopic samples. It was suggested to perform early stage nondestructive testing methods while the turbine rotor is in operation to avoid the rotor failure. Nikravesh, M. Y. et.al.[14] analyzed the propagation of a circumferential crack at three points of the turbine rotor. Through the obtained results, a crack front shape is achieved which can be used in rotor vibration analysis. Vasovic I. et. al. [15] have focused on the stress analysis and the determination of fracture mechanics parameters in low pressure (LP) turbine rotor discs and on developing analytic expressions for stress intensity factors at the critical location of LP steam turbine disc. They have used conventional finite elements to determine stress intensity factors. Shlyannikov, V. N. et. al. [16] determined the elastic-plastic fracture mechanics parameters, full-size stress- strain state analysis of the turbine disk was performed for semi-elliptical cracks under startup loading conditions. Prasad K. et.al. [17] performed experimental analysis on the forged turbine disc to study the effect of directionality on the crack growth behavior and observed that a higher crack growth rate in radial direction than tangential direction in a forged turbine disc made of a super alloy. Hu D. et.al. [18] discussed the possible microscopic mechanisms to explain the grain size effect on the FCG behaviors based on crack deflection and blockage, and the crack closure inducements involving plasticity. Hu D. et.al. [19]conducted tests on tension specimens and investigated the fatigue crack growth characteristics using stochastic fatigue crack growth model. Based on the experimental results, they performed probabilistic analysis on the damage tolerance of the turbine disk.

From the literature review, it is observed that most of the researchers have studied the effect of semi elliptical crack on different components like cylinders, pipes, plates, pressure vessels, rotors etc. using different approaches. Some of the researchers had concentrated on the effect of crack in turbine rotor. But the all the researchers have concentrated only on the experimental analysis of specimen at the failure zone of the turbine rotor after it failed. The objective of this paper is to present analytical approach to investigate the stress intensity factors of semi elliptical crack in the steam turbine rotor system.

\section{MODELLING OF TAPERED ROTATING DISK}

The stresses induced in rotors are three dimensional as they are of complex geometry. Hence in order to obtain the meaningful results, there is a need to have some assumption so that these assumptions simplify the complexity of the problem and lead to closed form solutions. One assumption that can be made is the axial symmetry with reference to the geometrical shape and also forces acting. Even though the symmetry related to geometry may not be justified completely, but the effect of disk uniformities can be considered later as localized effect .Another assumption that can be introduced is planes stress, if the dimensions along the axial direction are not too large. This type of rotating member is called as thin disc. With this assumption, the loads acting are considered on the plane $x y$ and the stresses acting along the $z$ direction can be considered as zero and the stress state is plane. 
The general governing differential equation Eq.(1) which represents a rotating disc along the radius is

$$
\begin{aligned}
& \frac{\mathrm{d}^{2} u}{\mathrm{~d} r^{2}}+\left(\frac{1}{t} \frac{\mathrm{d} h}{\mathrm{~d} r}+\frac{1}{r}\right) \frac{\mathrm{d} u}{\mathrm{~d} r}+\left(\frac{v}{t r} \frac{\mathrm{d} h}{\mathrm{~d} r}-\frac{1}{r^{2}}\right) u+ \\
& (1+v) \alpha\left(\frac{\mathrm{d} T}{\mathrm{~d} r}+\frac{T}{t} \frac{\mathrm{d} h}{\mathrm{~d} r}\right)+\left(1-v^{2}\right) \frac{\gamma \omega^{2} r}{E}=0
\end{aligned}
$$

For a conical disc as shown in Fig. 1, $\mathrm{t}=\mathrm{t}_{0}(1-\mathrm{k})$

where $t_{0}$ is the imaginary disc thickness at the axis, $k$ is dimensionless variable representing radius $r / R$

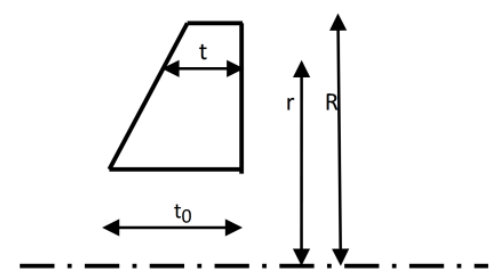

Fig. 1. Tapered rotating disc

To derive the standard equation for displacement field of a disc of varying thickness, the above relation is introduced in equation along with its derivatives w.r.t. t. and the simplified model is given in Eq.(2):

$$
\begin{gathered}
k^{2}(1-k) \frac{\mathrm{d}^{2} u}{\mathrm{~d} t^{2}}+k(1-2 k) \frac{\mathrm{d} u}{\mathrm{~d} t}-(1-k+v k) u=-C R^{3} k^{3}+C R^{3} k^{4} \\
\text { where } C=\frac{\left(1-v^{2}\right) \gamma \omega^{2}}{E}
\end{gathered}
$$

The solution of the above differential equation gives the radial displacement and the radial and tangential stresses induced given in Eq. (3)

$$
\begin{aligned}
& u=C_{1} q_{1}+C_{2} q_{2}+u_{p} \\
& \sigma_{r}=A a_{r}+B b_{r}+\sigma_{0} g_{r} \\
& \sigma_{t}=A a_{t}+B b_{t}+\sigma_{0} g_{t}
\end{aligned}
$$

Where

$$
\begin{gathered}
a_{r}=\frac{\mathrm{d} q_{1}}{\mathrm{~d} k}+J \frac{q_{1}}{k} \\
b_{r}=\frac{\mathrm{d} q_{2}}{\mathrm{~d} k}+J \frac{q u_{2}}{k} \\
a_{t}=\frac{q_{1}}{k}+J \frac{\mathrm{d} q_{1}}{\mathrm{~d} k} \text { and } b_{t}=\frac{q_{2}}{k}+J \frac{\mathrm{d} q_{2}}{\mathrm{~d} k} \\
g_{r}=\frac{-(3+v) t^{2}}{(11+v)}+\frac{(3+v)(2+v) t}{(5+v)(11+v)}+\frac{3(3+v)}{(5+v)(11+v)}
\end{gathered}
$$

To determine the constants $A$ and $B$, four different cases i.e. disc subjected to radial stress at inner radius, at outer radius, at inner and outer radii and only centrifugal load are considered and the expressions for stresses induced and radial displacement in the disc are determined.

\section{STRESSES IN STEAM TURBINE ROTOR}

Using the concept of superposition, the equations for applied to determine the stresses induced at blade mounting locations of a steam turbine rotor. The mechanical component as shown in Fig. 2 is the axisymmetric plane of a steam turbine rotor disc with radii at different sections as $a_{1}, a_{2}, a_{3}, a_{4}$ and $a_{5}$. The thicknesses at the corresponding sections are $b_{1}, b_{2}, b_{3}, b_{4}$ and $b_{5}$. The disc rotates with angular velocity of $2600 \mathrm{rpm}$. The surface forces acting at radii $a_{1}, a_{2}, a_{4}$ and $a_{5}$ are considered as zero and the only surface force considered is at radii $a_{3}$. This force is the centrifugal force due to the rotation of the blade. Using the above equations derived, the stresses as well as the radial displacement are determined.

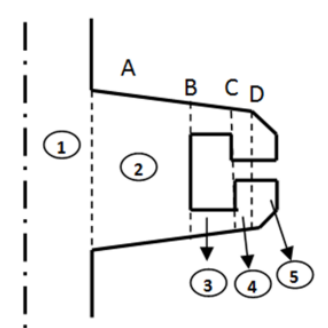

Fig. 2. Blade mounting location of turbine. rotor

To analyze this problem, first it is necessary to determine the stresses at radii $a_{1}, a_{2}, a_{3}$ and $a_{4}$ which are designated as $\sigma_{\mathrm{a} 1}, \sigma_{\mathrm{a} 2}, \sigma_{\mathrm{a} 3}$ and $\sigma_{\mathrm{a} 4}$. Hence there are four unknown stresses which are to be determined. For this the compatibility equations for radial displacement are considered i.e. at each radius, the radial displacement of the outer edge of inner part is equal to the radial displacement of inner edge of outer part. Again principle of superposition is used to determine the radial displacement at each interface.

At interface $A$ of radius $a_{1}$, the radial displacement considering the interface $A$ on zone 1 equated with the radial displacement considering the same interface $A$ on zone 2 to determine a linear equation with unknowns $\sigma_{a 1}$ and $\sigma_{a 2}$. The radial displacement of interface $A$ considering it on zone 1 is equal to the sum of two contributions 
determined at radius $\mathrm{a}_{1}$ ie radial displacement due to centrifugal force of zone 1 and stress $\sigma_{a 1}$.

The radial displacement of the same interface $A$ considering it on zone 2 is equal to the sum of three contributions again determined at radius $a_{1}$ i.e. radial displacement due to centrifugal force on zone 2 , stress $\sigma_{\mathrm{a} 1}$ and stress $\sigma_{\mathrm{a} 2}$. Applying the same compatibility condition at the remaining interfaces, four linear equations are obtained. These equations are solved and the stresses $\sigma_{\mathrm{a} 1}, \sigma_{\mathrm{a} 2}, \sigma_{\mathrm{a} 3}$ and $\sigma_{a 4}$ at the interfaces $A, B, C$ and $D$ are determined. The variation of radial stress the radius is shown in Fig. 3 .

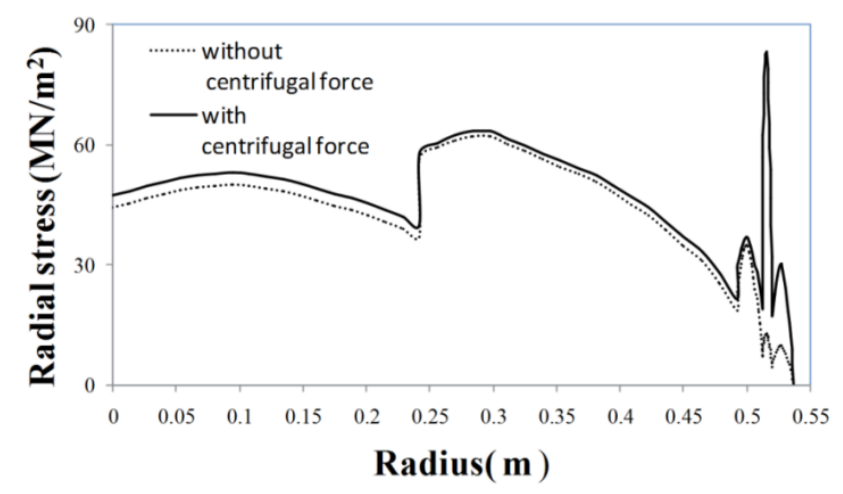

Fig. 3. Variation of radial stress

From the radial stress variation shown in Figure 3 , it is observed that the radial stresses induced are relatively higher with the consideration of blade rotation centrifugal force. Especially at the blade mounting locations, there is an instant increase in the radial stress by almost 4.5 times. This indicates the probability of Mode I type crack formation at this location. Hence, a semi elliptical crack is considered at the blade mounting location and the weight function approach is applied to determine the SIFs.

\section{WEIGHT FUNCTION APPROACH WITH PIECE WISE POLYNOMIAL FUNCTION}

Whenever cracks are subjected to arbitrary stress distribution, to evaluate the stress intensity factor, the weight function method is widely used. The high efficiency of this method is that the weight function depends only on the geometry of the crack. Once the weight functions are derived, then Mode I SIF can be determined using Eq. (4) given.

$$
K_{r}=\int_{0}^{a} \sigma_{r}(x) m(x, a) d x
$$

where $\sigma_{r}$ is the arbitrary stress distribution along the crack, $m(x, a)$ is Mode I weight function and $\mathrm{a}$ is the crack depth , $\mathrm{x}$ is the distance from the surface towards the crack tip. In the current problem, two different weight functions are assumed for surface and the deepest points.

A polynomial equation is used to represent the actual stress distribution along the crack length. The stresses at discrete points are obtained using the analytical approach given in the earlier section. The actual stress distribution is divided into $n$ number of intervals and the variation of stress between two arbitrary discrete points $x_{i}$ and $x_{i+1}$ is assumed as linear variation

$$
\begin{gathered}
\sigma_{i}(x)=A_{i}+B_{i} x, \mathrm{i}=0,1, \ldots \ldots ., \mathrm{n} \\
A_{i}=\sigma\left(x_{i}\right)-x_{i}\left[\frac{\sigma\left(x_{i+1}\right)-\sigma\left(x_{i}\right)}{x_{i+1}-x_{i}}\right] \text { and } \\
B_{i}=\frac{\sigma\left(x_{i+1}\right)-\sigma\left(x_{i}\right)}{x_{i+1}-x_{i}}
\end{gathered}
$$

where $A_{i}$ is the constant representing the actual stress distribution, $B_{i}$ is the coefficient representing the actual stress.

To determine the stress intensity factors, weight functions with five and six terms are considered separately and the corresponding equations are derived.

The five term weight function considered at the deepest point is given by Eq.(5).

$$
m(x, a)=\frac{2}{(2 \pi(a-x))^{\frac{1}{2}}}\left[\begin{array}{l}
1+M_{D 1}\left(1-\frac{x}{a}\right)^{\frac{1}{2}}+M_{D 2}\left(1-\frac{x}{a}\right) \\
+M_{D 3}\left(1-\frac{x}{a}\right)^{\frac{3}{2}}+M_{D 4}\left(1-\frac{x}{a}\right)^{2}
\end{array}\right]
$$

The corresponding stress intensity factor is given by Eq. (6)

$$
\begin{array}{r}
K_{I}=K_{W D 0}+K_{W D 1} M_{D 1}+K_{W D 2} M_{D 2}+ \\
K_{W D 3} M_{D 3}+K_{W D 4} M_{D 4}
\end{array}
$$

where

$$
\begin{aligned}
& K_{W D 0}=\int_{x_{i}}^{x_{i}+1} \sum_{i=1}^{n}\left(A_{i}+B_{i} x\right) \frac{2}{(2 \pi(a-x))^{\frac{1}{2}}} d x \\
&= \frac{2}{3} \sqrt{\frac{2}{\pi}} \sum_{i=1}^{i=1 n}\left[\begin{array}{l}
\sqrt{a-x_{i+1}}\left(-3 A_{i}-2 B_{i} a-B_{i} x_{i+1}\right) \\
+\sqrt{a-x_{i}}\left(3 A_{i}+2 B_{i} a+B_{i} x_{i}\right)
\end{array}\right] \\
& K_{W D 1}=\int_{x_{i}}^{x_{i}+1} \sum_{i=1}^{n}\left(A_{i}+B_{i} x\right) \frac{2}{(2 \pi(a-x))^{\frac{1}{2}}}\left(1-\frac{x}{a}\right)^{\frac{1}{2}} d x \\
&=\sqrt{\frac{2}{\pi}} \frac{1}{\sqrt{a}} \sum_{i=1}^{n}\left[A_{i}\left(x_{i+1}-x_{i}\right)+\frac{B_{i}}{2}\left(x_{i+1}^{2}-x_{i}^{2}\right)\right]
\end{aligned}
$$




$$
\begin{aligned}
K_{W D 2} & =\int_{x_{i}}^{x_{i+1}} \sum_{i=1}^{n}\left(A_{i}+B_{i} x\right) \frac{2}{(2 \pi(a-x))^{\frac{1}{2}}}\left(1-\frac{x}{a}\right) d x \\
& =\frac{2}{15} \frac{1}{\sqrt{a}} \sqrt{\frac{2}{\pi}} \sum_{i=1}^{n}\left[\begin{array}{l}
\left.\left(a-x_{i+1}\right)^{\frac{3}{2}}\left(-5 A_{i}-3 B_{i} x_{i+1}-2 a B_{i}\right)+\right] \\
\left(a-x_{i}\right)^{\frac{3}{2}}\left(5 A_{i}+3 B_{i} x_{i}+2 a B_{i}\right)
\end{array}\right] \\
K_{W D 3} & =\int_{x_{i}}^{x_{i+1}} \sum_{i=1}^{n}\left(A_{i}+B_{i} x\right) \frac{2}{(2 \pi(a-x))^{\frac{1}{2}}}\left(1-\frac{x}{a}\right)^{2} d x \\
& =\frac{2}{15} \frac{1}{\sqrt{a}} \sqrt{\frac{2}{\pi}} \sum_{i=1}^{n}\left[\begin{array}{l}
\left(a-x_{i+1}\right)^{\frac{3}{2}}\left(-5 A_{i}-3 B_{i} x_{i+1}-2 a B_{i}\right) \\
+\left(a-x_{i}\right)^{\frac{3}{2}}\left(5 A_{i}+3 B_{i} x_{i}+2 a B_{i}\right)
\end{array}\right] \\
K_{W D 4} & =\int_{x_{i}}^{x_{i+1}} \sum_{i=1}^{n}\left(A_{i}+B_{i} x\right) \frac{2}{\{2 \pi(a-x)\}^{\frac{1}{2}}}\left(1-\frac{x}{a}\right)^{\frac{5}{2}} d x \\
= & \frac{1}{35 a^{2}} \sqrt{\frac{2}{\pi} \sum_{i=1}^{n}}\left[\begin{array}{l}
\left(a-x_{i+1}\right)^{\frac{5}{2}}\left(-14 A_{i}-10 B_{i} x_{i+1}-4 a B_{i}\right) \\
+\left(a-x_{i}\right)^{\frac{5}{2}}\left(14 A_{i}+10 B_{i} x_{i}+4 a B_{i}\right)
\end{array}\right]
\end{aligned}
$$

To determine the weight function coefficients, influence coefficients for the SIF are used. The stress intensity factor in terms of influence coefficients is given by

$$
K_{I}=\sum_{j=0}^{3} A_{j} G_{j} \sqrt{\frac{\pi a}{Q}}
$$

where $Q=1+1.464\left(\frac{a}{c}\right)^{1.65}$

The above equations along with the pure uniform, linear, quadratic and cubic stress distributions gives four linear equations and the solution of these four linear equations gives the four coefficients $M_{D 1}, M_{D 2}, M_{D 3}$ and $M_{D 4}$ given by set of following equations.

$M_{D 1}=\frac{\pi}{\sqrt{2 Q}}\left(-32 G_{0}+384 G_{1}-972 G_{2}+660 G_{3}\right)-\frac{512}{35}$

$M_{D 2}=\frac{\pi}{\sqrt{2 Q}}\left(\frac{945}{4} G_{0}-\frac{21735}{8} G_{1}+6615 G_{2}-\frac{17325}{4} G_{3}\right)+54$

$M_{D 3}=\frac{\pi}{\sqrt{2 Q}}\left(-480 G_{0}+5280 G_{1}-12420 G_{2}+7920 G_{3}\right)-\frac{512}{7}$

$M_{D 4}=\frac{\pi}{\sqrt{2 Q}}\left(\frac{1155}{4} G_{0}-\frac{24255}{8} G_{1}+6930 G_{2}-\frac{17325}{4} G_{3}\right)+33$

The five term weight function considered at the surface point is given by Eq.(7).

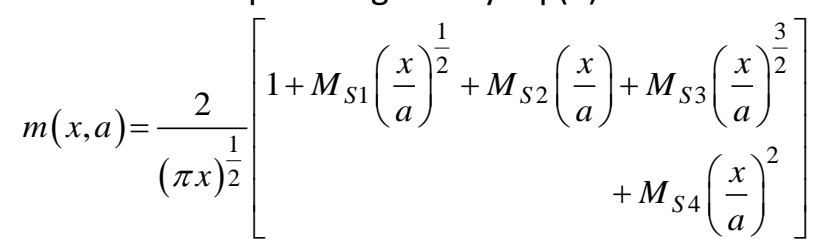

where $M_{i}$ are the weight function coefficients which depend on the geometry of the crack and the stress intensity factor is given by Eq. (8)

$$
\begin{gathered}
K_{I}=K_{W S 0}+K_{W S 1} M_{S 1}+K_{W S 2} M_{S 2}+K_{W S 3} M_{S 3} \\
+K_{W S 4} M_{S 4}
\end{gathered}
$$

where

$$
\begin{aligned}
& K_{W S 0}=\int_{x_{i}}^{x_{i}+1} \sum_{i=1}^{n}\left(A_{i}+B_{i} x\right) \frac{2}{(\pi x)^{\frac{1}{2}}} d x \\
& =\sqrt{\frac{16}{9 \pi}} \sum_{i=1}^{n}\left[\sqrt{x_{i+1}}\left(3 A_{i}+B_{i} x_{i+1}\right)-\sqrt{x_{i}}\left(3 A_{i}+B_{i} x_{i}\right)\right] \\
& K_{W S 1}=\int_{x_{i}}^{x_{i}} \sum_{i=1}^{n}\left(A_{i}+B_{i} x\right) \frac{2}{(\pi x)^{\frac{1}{2}}}\left(\frac{x}{a}\right)^{\frac{1}{2}} d x \\
& =\frac{1}{\sqrt{\pi a}} \sum_{i=1}^{n}\left[x_{i+1}\left(2 A_{i}+B_{i} x_{i+1}\right)-x_{i}\left(2 A_{i}+B_{i} x_{i}\right)\right] \\
& K_{W S 2}=\int_{x_{i}}^{x_{i}+1} \sum_{i=1}^{n}\left(A_{i}+B_{i} x\right) \frac{2}{(\pi x)^{\frac{1}{2}}}\left(\frac{x}{a}\right) d x \\
& =\frac{4}{15 a \sqrt{\pi}} \sum_{i=1}^{n} x_{i+1}^{\frac{3}{2}}\left(5 A_{i}+3 B_{i} x_{i+1}\right) x_{i}^{\frac{3}{2}}\left(5 A_{i}+3 B_{i} x_{i}\right) \\
& K_{W S 3}=\int_{x_{i}}^{x_{i}+1} \sum_{i=1}^{n}\left(A_{i}+B_{i} x\right) \frac{2}{(\pi x)^{\frac{1}{2}}}\left(\frac{x}{a}\right)^{2} d x \\
& =\frac{1}{3 a \sqrt{\pi a}} \sum_{i=1}^{n}\left[\begin{array}{c}
x_{i+1}^{2}\left(3 A_{i}+2 B_{i} x_{i+1}\right) \\
+x_{i}^{2}\left(3 A_{i}+2 B_{i} x_{i}\right)
\end{array}\right] \\
& K_{W S 4}=\int_{x_{i}}^{x_{i}+1} \sum_{i=1}^{n}\left(A_{i}+B_{i} x\right) \frac{2}{(\pi x)^{\frac{1}{2}}}\left(\frac{x}{a}\right)^{2} d x \\
& =\frac{1}{35 a^{2} \sqrt{\pi}} \sum_{i=1}^{n}\left[\begin{array}{c}
x_{i+1}^{\frac{5}{2}}\left(7 A_{i}+5 B_{i} x_{i+1}\right) \\
-x_{i}^{\frac{5}{2}}\left(7 A_{i}+5 B_{i} x_{i}\right)
\end{array}\right]
\end{aligned}
$$

To determine the weight function coefficients for the surface point, the same procedure adopted for the deepest point is used and the solution of four linear equations gives the coefficients

$$
\begin{aligned}
& M_{S 1}=\frac{\pi}{\sqrt{2 Q}}\left(40 G_{0}-420 G_{1}+1008 G_{2}-660 G_{3}\right)-\frac{512}{35} \\
& M_{S 2}=\frac{\pi}{\sqrt{2 Q}}\left(300 G_{0}-4200 G_{1}+11340 G_{2}-7920 G_{3}\right)-\frac{512}{7}
\end{aligned}
$$


$M_{S 3}=\frac{\pi}{\sqrt{2 Q}}\left(\frac{-1575}{8} G_{0}+\frac{19845}{8} G_{1}-\frac{25515}{4} G_{2}+\frac{17325}{4} G_{3}\right)+54 . \quad \begin{aligned} & \text { STRESS INTENSITY FACTORS AT BLADE } \\ & \text { MOUNTING LOCATIONS }\end{aligned}$ $M_{S 4}=\frac{\pi}{\sqrt{2 Q}}\left(\frac{-1155}{8} G_{0}+\frac{17325}{8} G_{1}-\frac{24255}{4} G_{2}+\frac{17325}{4} G_{3}\right)+3$ distribution, the probability of crack formation is

The six term weight function considered at the deepest point and surface points are given by Eq.(9) and Eq.(10).

$$
\begin{aligned}
& m(x, a)=\frac{2}{(2 \pi(a-x))^{\frac{1}{2}}}\left[\begin{array}{l}
1+M_{D 1}\left(1-\frac{x}{a}\right)^{\frac{1}{2}}+M_{D 2}\left(1-\frac{x}{a}\right)+ \\
M_{D 3}\left(1-\frac{x}{a}\right)^{\frac{3}{2}}+M_{D 4}\left(1-\frac{x}{a}\right)^{2} \\
+M_{D 5}\left(1-\frac{x}{a}\right)^{\frac{5}{2}}
\end{array}\right] \\
& m(x, a)=\frac{2}{(\pi x)^{\frac{1}{2}}}\left[\begin{array}{l}
1+M_{S 1}\left(\frac{x}{a}\right)^{\frac{1}{2}}+M_{S 2}\left(\frac{x}{a}\right)+M_{S 3}\left(\frac{x}{a}\right)^{\frac{3}{2}} \\
+M_{S 4}\left(\frac{x}{a}\right)^{2}+M_{S 5}\left(\frac{x}{a}\right)^{\frac{5}{2}}
\end{array}\right]
\end{aligned}
$$

Using the same procedure used for five term weight function, the equations for the coefficients are derived and given below.

$$
\begin{aligned}
& M_{D 1}=-\frac{13568}{805}+\frac{\pi}{3} \sqrt{\frac{2}{Q}}\left(-704 G_{0}+7818 G_{1}-18738 G_{2}+12210 G_{3}\right) \\
& M_{D 2}=\frac{1746}{23}+\frac{\pi}{184} \sqrt{\frac{2}{Q}}\left(\begin{array}{l}
48195 G_{0}-517860 G_{1}+ \\
1203930 G_{2}-762300 G_{3}
\end{array}\right) \\
& M_{D 3}=-\frac{22578}{161}+\frac{\pi}{23} \sqrt{\frac{2}{Q}\left(\begin{array}{l}
-15600 G_{0}+162780 G_{1} \\
-369630 G_{2}+229680 G_{3}
\end{array}\right)} \\
& M_{D 4}=\frac{2607}{23}+\frac{\pi}{184} \sqrt{\frac{2}{Q}}\left(\begin{array}{l}
123585 G_{0}-1261260 G_{1} \\
+2820510 G_{2}-1732500 G_{3}
\end{array}\right) \\
& M_{D 5}=\frac{-768}{23}+\frac{\pi}{23} \sqrt{\frac{2}{Q}}\left(\begin{array}{l}
-5040 G_{0}+51030 G_{1} \\
-113400 G_{2}+69300 G_{3}
\end{array}\right) \\
& \mathrm{M}_{\mathrm{S} 1}=-\frac{128}{7}+\frac{30 \pi}{\sqrt{\mathrm{Q}}}\left(\mathrm{G}_{0}-14 \mathrm{G}_{1}+42 \mathrm{G}_{2}-33 \mathrm{G}_{3}\right) \\
& \mathrm{M}_{\mathrm{S} 2}=90-\frac{315 \pi}{8 \sqrt{\mathrm{Q}}}\left(5 \mathrm{G}_{0}-84 \mathrm{G}_{1}+270 \mathrm{G}_{2}-220 \mathrm{G}_{3}\right) \\
& \mathrm{M}_{\mathrm{S} 3}=-\frac{1280}{7}+\frac{30 \pi}{\sqrt{\mathrm{Q}}}\left(15 \mathrm{G}_{0}-280 \mathrm{G}_{1}+945 \mathrm{G}_{2}-792 \mathrm{G}_{3}\right) \\
& M_{\mathrm{S} 4}=165-\frac{3465 \pi}{8 \sqrt{\mathrm{Q}}}\left(\mathrm{G}_{0}-20 \mathrm{G}_{1}+70 \mathrm{G}_{2}-60 \mathrm{G}_{3}\right) \\
& M_{S 5}=-\frac{384}{7}+\frac{30 \pi}{\sqrt{Q}}\left(5 G_{0}-105 G_{1}+378 G_{2}-330 G_{3}\right)
\end{aligned}
$$
high at the blade mounting location. Hence, a semi elliptical crack is modelled as shown in Fig. 4 and the SIFs are calculated using the above approaches. The SIFs for different crack depth ratios of 0.2 to 1 are shown in Fig. 5 and Fig. 6 for the surface and the deepest point with five term weight function and Fig. 7 and Fig. 8 for the surface and the deepest point with six term weight function respectively.

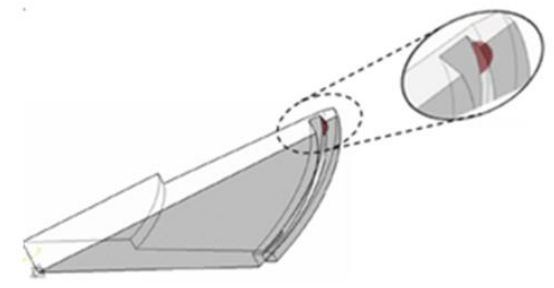

Fig. 4. Semi elliptical crack at blade mounting location

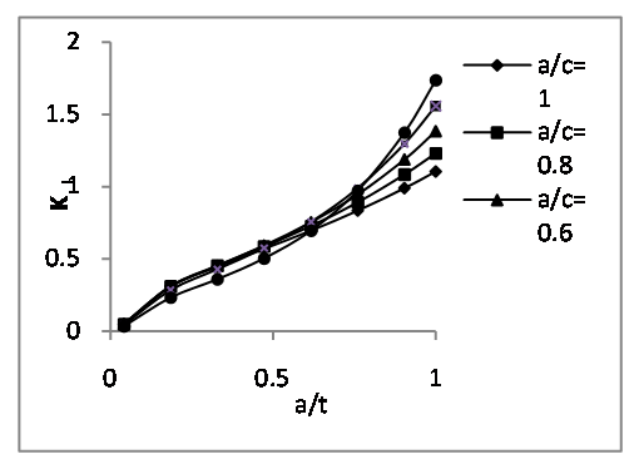

Fig. 5. Variation of SIF at the surface point using five term weight function

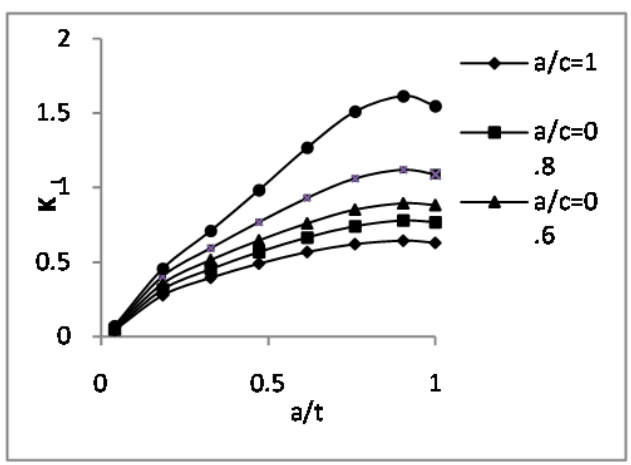

Fig. 6. Variation of SIF at the deepest point using five term weight function 


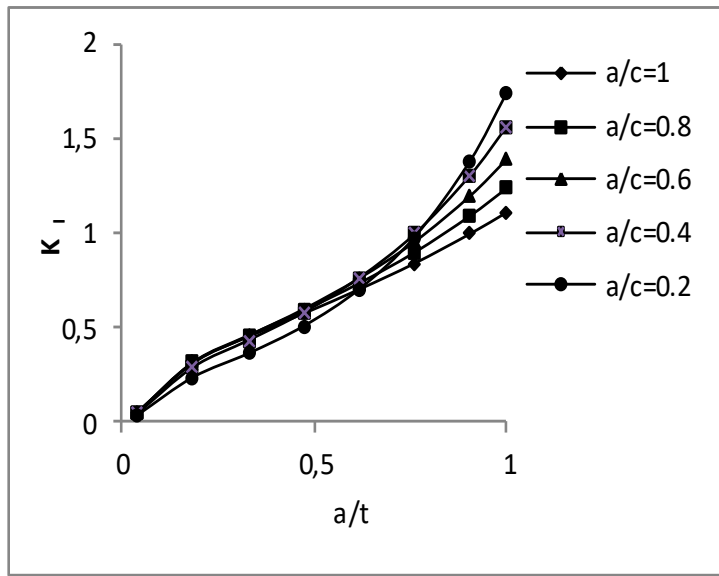

Fig. 7. Variation of SIF at the surface point using six term weight function.

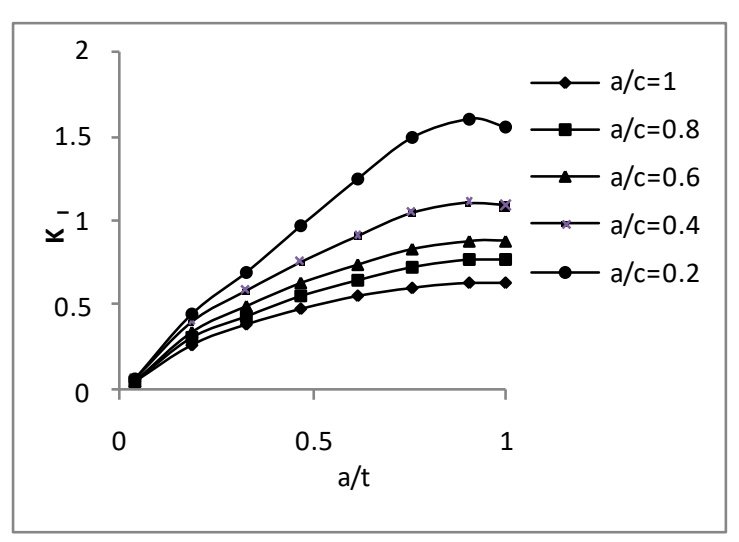

Fig. 8. Variation of SIF at the deepest point using six term weight function.

The SIF obtained using five term and six term weight function approach are validated with influence coefficient approach [5]. The SIFs at the surface and the deepest point using influence coefficient approach are presented in Fig. 9 and Fig. 10

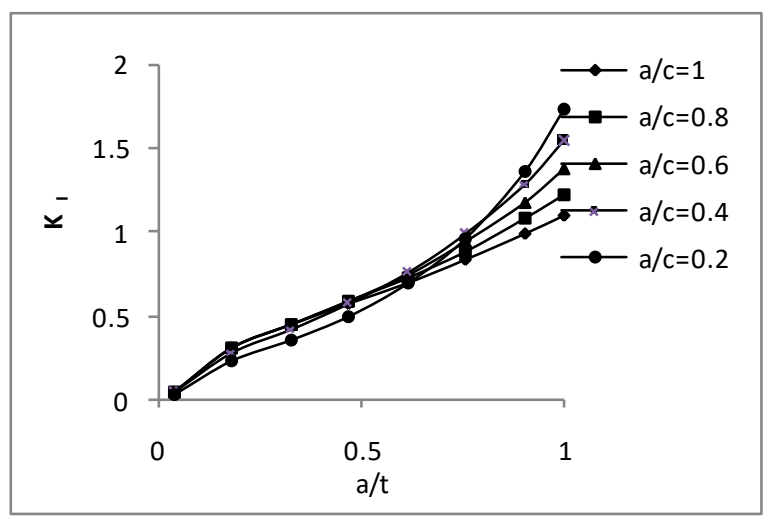

Fig 9. Stress intensity factor at deepest point using influence coefficient method

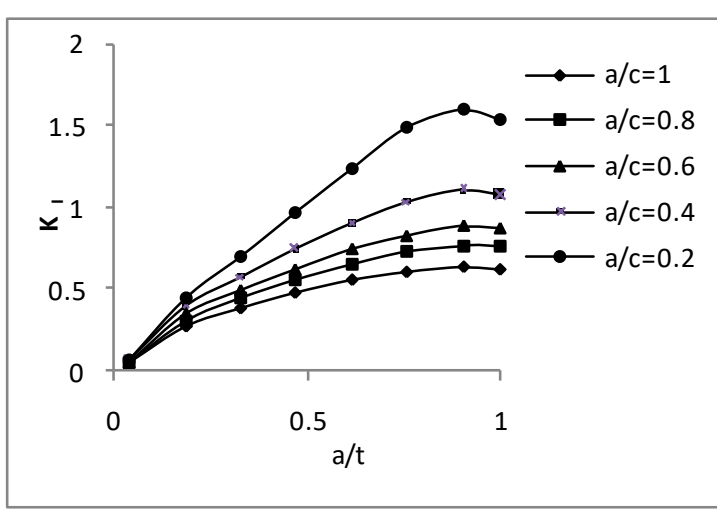

Fig 10. Stress intensity factor at surface point using influence coefficient method

The difference between five term and six term weight functions is presented in Table 1 and Table 2 for surface and deepest points. The accuracy improved for the proposed method w.r.t . the influence coefficient approach is presented in Table 3 and Table 4.

Table 1 . Difference (\%) in SIF at surface point obtained between five term and six term weight function

\begin{tabular}{|c|c|c|c|c|c|}
\hline \multirow{2}{*}{$\mathrm{a} / \mathrm{t}$} & \multicolumn{5}{|c|}{$\mathrm{a} / \mathrm{c}$} \\
\cline { 2 - 6 } & 1 & 0.8 & 0.6 & 0.4 & 0.2 \\
\hline 0.04 & 0.202 & 0.400 & 0.319 & 0.415 & 0.269 \\
\hline 0.184 & 0.514 & 0.609 & 0.394 & 0.769 & 0.815 \\
\hline 0.328 & 0.425 & 0.308 & 0.488 & 0.491 & 0.501 \\
\hline 0.472 & 0.263 & 0.256 & 0.304 & 0.208 & 0.337 \\
\hline 0.616 & 0.417 & 0.316 & 0.385 & 0.423 & 0.445 \\
\hline 0.76 & 0.252 & 0.224 & 0.222 & 0.202 & 0.216 \\
\hline 0.904 & 0.303 & 0.231 & 0.210 & 0.239 & 0.138 \\
\hline 1 & 0.181 & 0.122 & 0.130 & 0.071 & 0.012 \\
\hline
\end{tabular}

Table 2. Difference (\%) in SIF at deepest point obtained between five term and six term weight function

\begin{tabular}{|c|c|c|c|c|c|}
\hline \multirow{2}{*}{$\mathrm{a} / \mathrm{t}$} & \multicolumn{5}{|c|}{$\mathrm{a} / \mathrm{c}$} \\
\cline { 2 - 6 } & 1 & 0.8 & 0.6 & 0.4 & 0.2 \\
\hline 0.04 & 0.215 & 0.125 & 0.235 & 0.123 & 0.132 \\
\hline 0.184 & 0.095 & 0.124 & 0.123 & 0.190 & 0.125 \\
\hline 0.328 & 0.014 & 0.135 & 0.215 & 0.231 & 0.102 \\
\hline 0.472 & 0.086 & 0.012 & 0.096 & 0.020 & 0.012 \\
\hline 0.616 & 0.086 & 0.758 & 0.042 & 0.159 & 0.126 \\
\hline 0.76 & 0.239 & 0.154 & 0.099 & 0.179 & 0.012 \\
\hline 0.904 & 0.212 & 0.249 & 0.321 & 0.289 & 0.158 \\
\hline 1 & 0.132 & 0.124 & 0.125 & 0.046 & 0.214 \\
\hline
\end{tabular}


Table 3. Accuracy improved (\%) in SIF at surface point w.r.t influence coefficient method

\begin{tabular}{|c|c|c|c|c|c|}
\hline \multirow{2}{*}{$\mathrm{a} / \mathrm{t}$} & \multicolumn{5}{|c|}{$\mathrm{a} / \mathrm{c}$} \\
\cline { 2 - 6 } & 1 & 0.8 & 0.6 & 0.4 & 0.2 \\
\hline 0.04 & 3.4743 & 3.6784 & 3.6625 & 3.8386 & 4.2794 \\
\hline 0.184 & 2.3094 & 2.0694 & 2.2430 & 2.4014 & 3.2701 \\
\hline 0.328 & 1.3729 & 1.4872 & 1.4502 & 1.5286 & 1.7682 \\
\hline 0.472 & 0.9869 & 0.9598 & 1.0164 & 0.9574 & 1.2168 \\
\hline 0.616 & 1.1546 & 1.0192 & 1.0921 & 1.1543 & 1.2686 \\
\hline 0.76 & 0.7820 & 0.7310 & 0.7089 & 0.6794 & 0.7216 \\
\hline 0.904 & 0.8216 & 0.7123 & 0.5645 & 0.6493 & 0.4227 \\
\hline 1 & 0.5721 & 0.4718 & 0.4344 & 0.2634 & 0.0979 \\
\hline
\end{tabular}

Table 4. Accuracy improved (\%) in SIF at deepest point w.r.t influence coefficient method

\begin{tabular}{|c|c|c|c|c|c|}
\hline \multirow{2}{*}{$\mathrm{a} / \mathrm{t}$} & \multicolumn{5}{|c|}{$\mathrm{a} / \mathrm{c}$} \\
\cline { 2 - 6 } & 1 & 0.8 & 0.6 & 0.4 & 0.2 \\
\hline 0.04 & 1.5678 & 1.8102 & 2.3259 & 0.8512 & 1.1510 \\
\hline 0.184 & 2.2629 & 2.9044 & 2.6586 & 2.9450 & 3.0190 \\
\hline 0.328 & 3.6585 & 4.0248 & 3.7767 & 3.3516 & 3.8775 \\
\hline 0.472 & 4.0011 & 4.0289 & 3.9378 & 3.6370 & 2.5346 \\
\hline 0.616 & 3.4399 & 4.9523 & 3.8596 & 3.7667 & 3.2927 \\
\hline 0.76 & 3.5677 & 3.3845 & 2.4444 & 2.9107 & 3.0911 \\
\hline 0.904 & 2.6164 & 2.2198 & 2.4582 & 3.0288 & 2.2444 \\
\hline 1 & 0.9705 & 0.0864 & 0.8491 & 0.5293 & 0.2043 \\
\hline
\end{tabular}

\section{CONCLUSIONS}

The present work is focussed on the analytical approach to determine the stress intensity factors at blade mounting locations in steam turbine rotor system. For this purpose, weight function approach is used and the stress intensity factors are determined at critical points surface and deepest points of semi elliptical crack. Two different weight functions with five and six term are used in this approach. The variation of SIF for crack depth ratio $\mathrm{a} / \mathrm{t}$ is determined for different crack ellipticity ratio a/c values. It is observed that as the crack depth ratio increases, the SIF values also increases for surface and deepest points. But for deepest points at higher crack ellipticity ratios, there is marginal decrease in the SIF value at higher crack depth ratio. The results obtained are compared with the influence approach and it is observed that there is improvement in the accuracy of the results by considering six term weight function.

\section{REFERENCES}

[1] J. Chen, H. Pan, Stress Intensity Factor of Semi-Elliptical Surface Crack in a Cylinder with Hoop Wrapped Composite Layer.
International Journal of Pressure Vessels and Piping, 110, 2013: 77-81.

http://doi.org/10.1016/i.ijpvp.2013.04.026

[2] J. Predan, V. Močilnik, N. Gubeljak, Stress Intensity Factors for Circumferential SemiElliptical Surface Cracks in a Hollow Cylinder Subjected To Pure Torsion. Engineering Fracture Mechanics, 105, 2013: 152-168.

http://doi.org/10.1016/j.engfracmech.2013.03.033

[3] M. K. Ramezani, J. Purbolaksono, A. Andriyana, S. Ramesh, I. S. Putra, Empirical Solutions for Stress Intensity Factors of a Surface Crack in a Solid Cylinder Under Pure Torsion. Engineering Fracture Mechanics, 193, 2018: 122-136.

http://doi.org/10.1016/i.engfracmech.2018.02.015

[4] X. Y. Zhang X. F. Li, Transient Thermal Stress Intensity Factors for a Circumferential Crack in a Hollow Cylinder Based on Generalized Fractional Heat Conduction. International Journal of Thermal Sciences, 121, 2017: 336347.

http://doi.org/10.1016/i.ijthermalsci.2017.07.015

[5] J. M. Alegre, I. I. Cuesta, Stress Intensity Factor Equations for Internal Semi-Elliptical Cracks in Pressurized Cylinders. Journal of Pressure Vessel Technology, 133 (5), 2011: No.054501. http://doi.org/10.1115/1.4002613

[6] A. R. Shahani, M. M. Shodja, A. Shahhosseini, Experimental Investigation and Finite Element Analysis of Fatigue Crack Growth in Pipes Containing a Circumferential Semi-Elliptical Crack Subjected to Bending. Experimental Mechanics, 50 (5), 2010: 563-573.

http://doi.org/10.1007/s11340-009-9229-6

[7] A. Zareei, S. M. Nabavi, Calculation Of Stress Intensity Factors for Circumferential SemiElliptical Cracks With High Aspect Ratio in Pipes. International Journal of Pressure Vessels and Piping, 146, 2016: 32-38.

http://doi.org/10.1016/j.ijpvp.2016.05.008

[8] D. Li, H. Yang, Y. Lu, Engineering Numerical Analysis of SIF and Security Service Evaluation on Thin- Walled Pipeline' S Semi-Elliptical Crack in NPS Under Heat-Stress Coupling Load. Procedia Engineering, 27, 2012: 15821587.

http://doi.org/10.1016/i.proeng.2011.12.624

[9] A. Benhamena, B. B. Bouiadjra, A. Amrouche, G. Mesmacque, N. Benseddiq, Three Finite Element Analysis of Semi-Elliptical Crack in High Density Poly-Ethylene Pipe Subjected to Internal Pressure. Materials and Design, 31 (6), 2012: 3038-3043.

http://doi.org/10.1016/i.matdes.2010.01.029 
[10] M. Banaszkiewicz, Numerical Investigations Of Crack Initiation in Impulse Steam Turbine Rotors Subject to Thermo-Mechanical Fatigue. Applied Thermal Engineering, 138, 2017: 761773.

http://doi.org/10.1016/i.applthermaleng.2018.04.099

[11] D. G. Hattingh, M. N. James, M. Newby, R. Scheepers, P. Doubell, Damage Assessment And Refurbishment of Steam Turbine Blade/Rotor Attachment Holes. Theoretical and Applied Fracture Mechanics, 83, 2016: 125-134.

http://doi.org/10.1016/i.tafmec.2015.11.001

[12] M. Banaszkiewicz, A. Rehmus-Forc, Stress Corrosion Cracking of A 60MW Steam Turbine Rotor. Engineering Failure Analysis, 51, 2015: 55-68.

http://doi.org/10.1016/i.engfailanal.2015.02.015

[13] S. Barella, M. Bellogini, M. Boniardi, S. Cincera, Failure Analysis of A Steam Turbine Rotor. Engineering Failure Analysis, 18 (6), 2011: 1511-1519.

http://doi.org/10.1016/i.engfailanal.2011.05.006

[14] M. Y. Nikravesh, M. Meidan Sharafi, Failure of a Steam Turbine Rotor Due to Circumferential Crack Growth Influenced by Temperature and Steady Torsion. Engineering Failure Analysis, 66, 2016: 296-311.

[15] I. Vasovic, S. Maksimovic, K Maksimovic, S. Stupar, G. Bakic, M. Maksimovic,
Determination of Stress Intensity Factors in Low Pressure Turbine Rotor Discs. Mathematical Problems in Engineering, 2014: ID 304638.

http://dx.doi.org/10.1155/2014/304638

[16] V. N. Shlyannikov, A. P. Zakharov, R. R. Yarullin, Structural Integrity Assessment of Turbine Disk on a Plastic Stress Intensity Factor Basis. International Journal of Fatigue, 92, 2016: 234-245.

http://doi.org/10.1016/i.ijfatigue.2016.07.016

[17] K. Prasad, N. C. Babu, V. Kumar, Effect of Frequency and Orientation on Fatigue Crack Growth Behavior of Forged Turbine Disc of IN 718 Superalloy. Materials Science and Engineering A, 544, 2012: 83-87.

http://doi.org/10.1016/i.msea.2012.02.088

[18] D. Hu, J. Mao, J. Song, F. Meng, X. Shan, R. Wang, Experimental Investigation of Grain Size Effect on Fatigue Crack Growth Rate in Turbine Disc Superalloy GH4169 Under Different Temperatures. Materials Science and Engineering $A, 669,2016$ : 318-331.

http://doi.org/10.1016/j.msea.2016.05.063

[19] D. Hu, R. Wang, J. Fan, X. Shen, Probabilistic Damage Tolerance Analysis on Turbine Disk Through Experimental Data. Engineering Fracture Mechanics, 87, 2012: 73-82.

http://doi.org/10.1016/j.engfracmech.2012.03.008 